\title{
阴离子与非离子表面活性剂复配体系反胶团的电导研究
}

\author{
刘金彦 * 韩外慧张 燕 姚超怀 \\ (内蒙古科技大学化学与化工学院, 内蒙古包头 014010)
}

\begin{abstract}
摘要：在 SDS/Tween60/正己醇/环已烷/水形成的反胶团复配体系中, 电导率 $(\kappa)$ 与水和表面活性剂的摩尔比 $\left(W_{0}\right)$ 关系曲线上存在最大值, 随着复配体系中 SDS 的摩尔分数 $\left(x_{\mathrm{SDS}}\right)$ 增大, 最大增溶水量 $\left(W_{0, \max }\right)$ 向 $W_{0}$ 值更大的方 向移动. $x_{\mathrm{SDS}} \leqslant 0.5$ 时, 随着 $x_{\mathrm{SDS}}$ 的增大, $W_{0, \text { max }}$ 所对应的电导率值增大; $x_{\mathrm{SDS}} \geqslant 0.5$ 时, 其电导率值减小. 在 AOT/ Tween60/环己烷/水体系中, 出现了与 SDS/Tween60/正己醇/正己烷/水体系类似的现象, 但 $W_{0, \max }$ 所对应的电导率 值, 随着 $x_{\text {AOT }}$ 的增大而增大, 不会出现极大值, 两者的差异主要是由于助表面活性剂醇的影响. 在 SDS/TritionX100/正己醇/环己烷/水体系中也印证了该结论.
\end{abstract}

关键词：电导; 复配体系; 反胶团; 增溶; 非离子表面活性剂 中图分类号: 0648

\section{Studies on the Conductivity of Mixed Reverse Micelles of Anionic and Non-Ionic Surfactants}

\author{
LIU Jin-Yan* HAN Wai-Hui Z ZHANG Yan YAO Chao-Huai \\ (College of Chemistry and Chemical Engineering, Inner Mongolia University of Science and Technology, \\ Baotou 014010, Inner Mongolia, P. R. China)
}

\begin{abstract}
In the SDS/Tween60/hexanol/cyclohexane/water system, a conductivity maximum was observed in the conductivity $(\kappa)$ and molar ratio of water to surfactants $\left(W_{0}\right)$ curves for mixed reverse micelles. The $W_{0, \max }$ moved to a bigger $W_{0}$ value as the $x_{\mathrm{SDS}}$ (molar fraction of anionic surfactant) value increased. At $x_{\mathrm{SDS}} \leqslant 0.5$, the conductivity values that correspond with $W_{0, \max }$ increased as $x_{\mathrm{SDS}}$ increased; and at $x_{\mathrm{SDS}} \geqslant 0.5$, the conductivity values decreased. In the AOT/ Tween60/cyclohexane/water system, the observed phenomenon was similar to the SDS/Tween60/hexanol/cyclohexane/ water system. However, conductivity values that correspond with $W_{0, \max }$ increased with increasing $x_{\text {AOT }}$ and there was no maximum value. The difference is mainly due to cosurfactant effects, which was confirmed by the SDS/TritionX-100/ hexanol/cyclohexane/water system.
\end{abstract}

Key Words: Conductivity; Mixed system; Reverse micelles; Solubilization; Non-ionic surfactant

表面活性剂在水和助表面活性剂存在的情况 下, 非极性溶剂中会自发聚集成反胶团, 其中水可以 形成水核, 电导法能够测定电导率随增溶水量的变 化关系, 可以间接得到反胶团结构的信息 ${ }^{[1-2]}$. 目前 人们对普通表面活性剂反胶团的性质已经作了大量 的研究, 现在的工作基本集中在其应用上, 即作为微
反应器 ${ }^{[3-4]}$ 、合成纳米粒子 ${ }^{[5-8]}$ 、蛋白质分离和萃取 ${ }^{[9]}$ 等 应用. 而且研究最多的表面活性剂是带两根烷烃链 的阴离子表面活性剂二(2-乙基己基)琥珀酸酯磺酸 钠(AOT $)^{[10-11]}$, 它不需要助表面活性剂就可以自发形 成反胶团, 但是对于普通表面活性剂形成的反胶团 所表现出来的不同于 AOT 体系的特殊的性质却报

Received: December 7, 2009; Revised: January 25, 2010; Published on Web: April 9, 2010.

*Corresponding author. Email: liujinyan2007@163.com; Tel:+86-15924426433.

The project was supported by the Items of Institution of Higher Education Scientific Research of Inner Mongolia, China (NJZY07104) and Natural Science Foundation of Inner Mongolia, China (20080404MS0210).

内蒙古高等学校科学研究项目(NJZY07104)和内蒙古自然科学基金(20080404MS0210)资助

C. Editorial office of Acta Physico-Chimica Sinica 
道甚少. 本文将十二烷基硫酸钠(SDS)与普通非离子 表面活性剂复配, 发现该体系与 AOT与非离子表面 活性剂复配体系所形成反胶团性质的不同, 解释了 其产生的原因, 为表面活性剂作为反胶团的应用奠 定了理论基础.

\section{1 实验部分}

\section{1 试剂与仪器}

二(2-乙基己基)琥珀酸酯磺酸钠(AOT, 分析纯, Sigma, 美国); 十二烷基硫酸钠(SDS, 分析纯, Farco Chemical Supplies, 香港); 吐温 60(Tween60, 分析纯, Sigma, 美国), 曲拉通-100(TritionX-100, 分析纯, Fluka, 瑞士); 环己烷(分析纯, 天津市华东试剂厂); 正己醇、 正丁醇(分析纯, 天津市化学试剂三厂).

SLDS-I 型数显电导率仪(南京桑力电子设备厂), DC0506 型精密控温槽(上海衡平仪器仪表厂), 控温 精度 $\pm 0.1{ }^{\circ} \mathrm{C}$, 实验温度 $30{ }^{\circ} \mathrm{C}$, 实验用水为 Milli-Q 水.

\section{2 实验方法}

将测定体系中的表面活性剂加人正己醇/环己 烷(体积比为 0.15:0.85)混合溶剂中, 使其浓度为 0.1 $\mathrm{mol} \cdot \mathrm{L}^{-1}$, 加超纯水, 快速混匀, 若溶液澄清透明, 在 $30{ }^{\circ} \mathrm{C}$ 下放置一定时间, 测其电导率值. 记录电导率 值和增溶水量 $\left(W_{0}\right)$, 画出两者的关系图, 其中

$$
W_{0}=\frac{[\text { water }]}{\text { [surfactant }]} \text {. }
$$

\section{2 结果与讨论}

\section{$2.1 \mathrm{SDS} /$ 正己醇/环己烷/水体系}

图 1 为 SDS 在环己烷和正己醇混合溶剂中的 电导率 $(\kappa)$ 随 $W_{0}$ 的变化关系, 电导率先随 $W_{0}$ 的增大 而增大, 达到极大值 $\left(W_{0}=W_{0, \text { max }}\right)$ 后则随 $W_{0}$ 的增大而 下降. 与文献中所报道曲线一致 ${ }^{[1-2]}$, 在AOT 与二(2-乙 基己基)磷酸(HDEHP)的复配体系在庚烷中电导率 随 $W_{0}$ 变化关系中也出现了上述现象, 主要是由于 电荷迁移引起. 当增溶水量较小时, 增溶的水分子主 要使表面活性剂头基离子发生水化, 与 $\mathrm{Na}^{+}$离子和 表面活性剂的头基结合.一些 $\mathrm{Na}^{+}$离子仍被束缚在 表面活性剂上, 不能移动, 这样使整个水环境基本静 止不动. 两个表面活性剂分子聚集在一起, 形成寿命 较短的二聚体, 然后彼此分开, 形成两个孤立的粒 子. 在这一过程中, 反离子随机的再分配, 在电场力 的作用下移人油相, 所以电导率较低. 电导率出现最

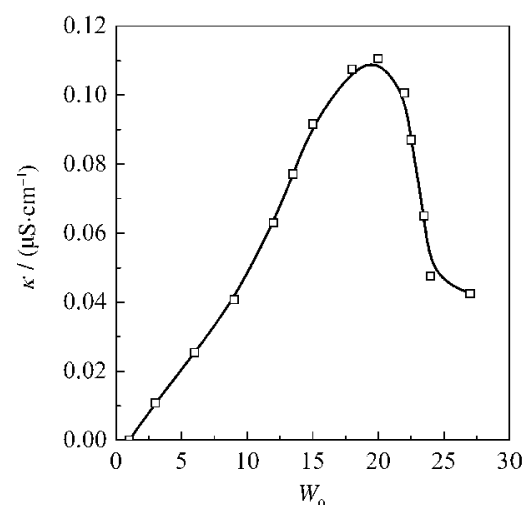

图 $1 \mathrm{SDS}$ /正己醇/环己烷/水体系的导电率 $(\kappa)-W_{0}$ 关系图

Fig.1 Variation of conductivity $(\kappa)$ with $W_{0}$ in SDS/ hexanol/cyclohexane/water system $W_{0}=[$ water $] /[$ surfactant $]$

大值是体系中两个相反因素共同作用的结果, 一方 面, 当水的浓度超过表面活性剂头基和反离子结合 的水时, 加人的水形成了在反胶团中的水核, 随着增 溶水量的增加, 在粒子发生碰撞和波动过程中, 水化 的反离子发生交换和再分配, 使电导率增加. 另一方 面, 反胶团水核的大小与其增溶水量呈线性关系, 粒 子半径的增加使其易于在反胶团的油水界面形成双 电层, 使粒径较大的带电粒子在电场力作用下移动 缓慢. 随着增溶水量的增加, 使电导率增加和减少的 因素同时起作用, 导致在电导率随增溶水量变化关 系中, 电导率出现最大值. 增溶水量继续增大, 反胶 团的水核增大, 带电粒子移动缓慢. 因此随着增溶水 量增加, 电导率降低.

\subsection{SDS/Tween60/正己醇/环己烷/水体系}

图 2 为阴离子表面活性剂 SDS 和 Tween60 复 配体系在正己醇/环己烷混合溶剂中的电导率随增

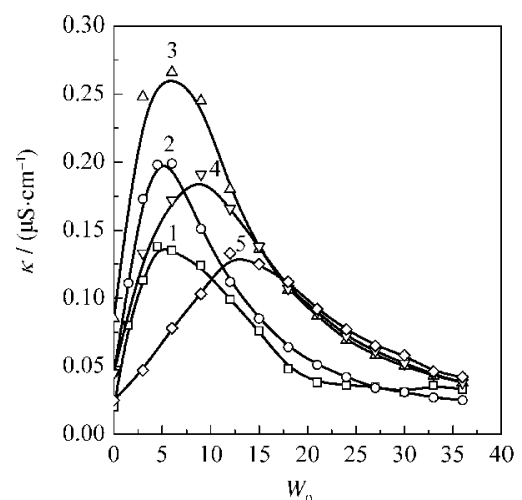

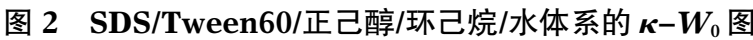

Fig.2 Variations of $\kappa$ with $W_{0}$ in SDS/Tween60/ hexanol/cyclohexane/water system $x_{\mathrm{SDS}}:$ (1) 0.1, (2) 0.3, (3) 0.5, (4) 0.7, (5) 0.9

$x_{\mathrm{SDS}}:$ molar fraction of SDS in SDS/Tween60/cyclohexane/water system 
溶水量的变化关系, 可见在复配体系中 SDS 所占的 摩尔分数 $\left(x_{\mathrm{SDS}}\right)$ 不同时, 电导率随增溶水量的变化关 系与图 1 一样, 即电导率先随 $W_{0}$ 的增大而增大, 达 到极大值 $\left(W_{0}=W_{0, \text { max }}\right)$ 后则随 $W_{0}$ 的增大而下降. 产生 此现象的原因与前述一致. 但是不同配比下的 $W_{0, \text { max }}$ 和其对应的电导率值却出现了如图 3 所示的变化关 系. $W_{0, \text { max }}$ 随着 $x_{\mathrm{SDS}}$ 增大而向 $W_{0}$ 增大的方向移动. 当 $x_{\mathrm{SDS}} \leqslant 0.5$ 时, 其对应的电导率值却呈现出随着增溶 水量的增大而增大, 当 $x_{\mathrm{SDS}} \geqslant 0.5$ 时, 随着增溶水量 的增大而减小.

Kallay 等 ${ }^{[2]}$ 推导出反胶团的电导率与粒子半径 $r$ 和界面层厚度 $l$ 的关系, $\mathrm{Ma}$ 等 ${ }^{[2]}$ 将其进行进一步化 简可以得出

$$
r=\frac{3}{2} l \text {, 可以记为 } r_{\text {max }}=\frac{3}{2} l
$$

(1) 式中 $r_{\text {max }}$ 为电导率最大时反胶团粒子的半径, $l$ 为 反胶团界面层厚度. 式(1)的物理意义为当表面活性 剂的浓度固定时, 反胶团达到最大电导率时, 其液滴 半径为 $3 / 2$ 倍的界面层厚度.

可以由式(1)得知, 所有导致粒子半径增大的因 素均会使反胶团的粒子半径在较小 $W_{0}$ 时达到最大 值, 即所有增大粒子半径的因素都会使 $W_{0, \max }$ 向更 小的 $W_{0}$ 值移动. Tween60 与 SDS 复配体系, 在 SDS 所形成的反胶团中, 逐渐加人 Tween60, 随着体系中 Tween60 含量的增加, 使反胶团粒子的半径增大, 使 $W_{0, \text { max }}$ 向增溶水量小的方向移动. 文献[9]中光散射结 果也证明了上述结论. 在阴离子表面活性剂 AOT 在庚烷中形成的反胶团中加人非离子表面活性剂 Brij30, 随着 Brij30 的加人量增加, 反胶团的粒子半 径增大. 结合式(1)可以得出使反胶团粒子半径增大 的因素, 均会使 $W_{0, \text { max }}$ 向增溶水量减小的方向移动.

从图 2 中还可以看出, 当 $x_{\mathrm{SDS}} \leqslant 0.5$ 时, 在整个 研究的 $W_{0}$ 范围内, 电导率随 $x_{\mathrm{SDS}}$ 的增大而增大. Eicke 和 Thomas ${ }^{[14]}$ 的研究表明, 在反胶团体系中, 尽 管阴离子表面活性剂主体和反离子都是电荷载体, 但只有反离子的充分移动对导电起作用. 如果反离 子 $\mathrm{Na}^{+}$能够完全电离, 应该是电导率值随着体系中 SDS 的摩尔分数增加而增大, 如图 2 中 $x_{\mathrm{SDS}} \leqslant 0.5$ 时 的电导率值. 体系中非离子表面活性剂 Tween 60 占 主要成分, 少量的 SDS 加人体系中形成反胶团, 其 头基完全电离, 所以体系的电导率随着 $x_{\mathrm{SDD}}$ 增大而 增大, 而与反胶团的增溶水量基本无关.

但是当 $x_{\mathrm{SDS}} \geqslant 0.5$ 时, 却出现了较为复杂的现象.
当增溶水量较小时, 以 $W_{0}=5$ 时为例, 随着 $x_{\mathrm{SDS}}$ 增加, 体系的电导率值减小. 当增溶水量较大时, 以 $W_{0}=25$ 为例, 电导率值随着体系中 $x_{\mathrm{SDS}}$ 的增大而增大. 如上 所述, 非离子表面活性剂的加人, 会使反胶团的粒子 半径增大, 在反胶团的界面层易于形成双电层, 使粒 径较大的带电粒子在电场力作用下移动缓慢, 虽然 反胶团粒子半径增大也有利于其发生电离, 但前者 为主要影响因素, 所以其电导率随着 $x_{\mathrm{SDS}}$ 的增大而 减小. 当增溶水量较大时, 在反胶团体系中随着增溶 水量的增大, 粒子半径增大, 虽然半径增大导致带电 粒子移动缓慢, 但表面活性剂的反离子能够发生充 分电离.

但上述解释均没有证明图 3 中 a 曲线所出现的 现象, 即最大增溶水量所对应电导率值在 $x_{\mathrm{SDS}}=0.5$ 时出现极大值, 我们与不需要加人醇作为助表面活 性剂的 AOT 体系做对比, 以期对该现象作出合理 的解释.

\subsection{AOT/Tween60/环己烷/水体系}

与上述体系对比, 我们研究了阴离子表面活性 剂为 AOT 时, 与 Tween60 复配在环己烷中的电导 行为, 如图 4 所示.

在 AOT/Tween60/环己烷体系中不需要加人助 表面活性剂正已醇, 表面活性剂在非极性溶剂中聚 集生成反胶团, 其几何排列因子 $P=V / S l_{\mathrm{c}}^{[15]}$ 应大于 1 , 其中 $V$ 和 $l_{\mathrm{c}}$ 分别为疏水碳氢链的体积和长度, $S$ 为 表面活性剂分子的头基面积. AOT 由于具有双烷烃 链, 满足此条件.

由图 4 可见, 随着 $x_{\mathrm{AOT}}$ 增大, $W_{0, \max }$ 值增大, 当增 溶水量达到 $W_{0, \text { max }}$ 时, 随着 $x_{\mathrm{AOT}}$ 增大, 其电导率值也 增大. 当 $W_{0}$ 值较小时, 电导率值随着 $x_{\mathrm{AOT}}$ 的增大而

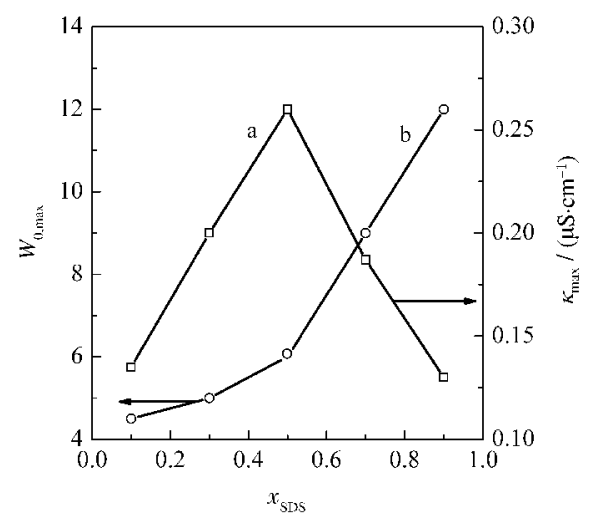

图 $3 \mathrm{SDS} / \mathrm{Tween} 60$ /正己醇/环己烷/水体系中 $\kappa_{\max }(\mathrm{a})$ 及 $W_{0, \max }(\mathrm{b})$ 随 $x_{\mathrm{SDs}}$ 变化关系

Fig.3 Variations of $\kappa_{\max }(\mathrm{a})$ and $W_{0, \max }$ (b) with $x_{\mathrm{SDS}}$ in SDS/Tween60/hexanol/cyclohexane/water system 


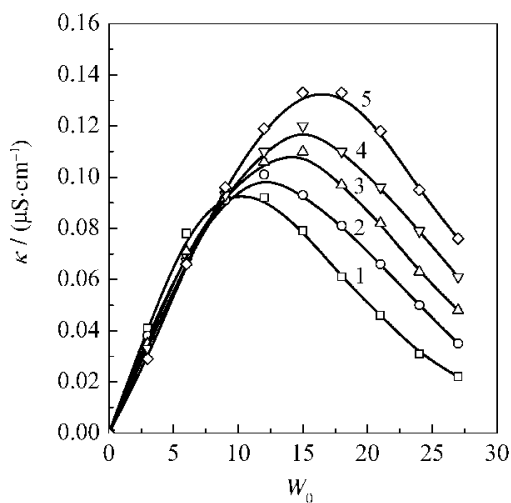

图 $4 \mathrm{AOT} / \mathrm{Tween} 60 / /$ 环己烷/水体系的 $\boldsymbol{\kappa}-\boldsymbol{W}_{0}$ 图

Fig.4 Variations of $\kappa$ with $W_{0}$ in AOT/Tween60/ cyclohexane /water system

$x_{\text {AOT: }}$ (1) 0.1, , (2) 0.3, (3) 0.5 , (4) 0.7 , (5) 0.9

减小, 而当 $W_{0}$ 值较大时, 电导率随着 $x_{\mathrm{AOT}}$ 的增大而 增大.

上述现象与 SDS/Tween60/环已烷/水体系类似. 但不同的是, 当阴离子表面活性剂的摩尔分数超过 0.5 时, 最大增溶水量所对应的电导率值却随着 $x_{\mathrm{AOT}}$ 的增大而增大, 而在 SDS 体系中其值却减小.

当体系中增溶水量足够大时, 我们假设表面活 性剂分子都排列在反胶团的油水界面上, AOT 与 SDS 的浓度一样, 说明排列在界面上的分子数一样, 但是在 SDS 体系中, 为了能够形成反胶团, 其界面 上有正己醇插人来降低其平均头基面积, SDS 的头 基面积与 $\mathrm{AOT}$ 的基本一样, 分别为 $0.53 \mathrm{~nm}^{2}[16]$ 和 $0.54 \mathrm{~nm}^{2[17]}$. 如果表面活性剂分子全部参与形成反胶 团, 在没有正己醇的情况下, SDS 和 AOT 体系形成 的反胶团的大小应一样, 但是 SDS 体系必须加人正 己醇来帮助形成反胶团, 尽管正己醇的头基面积很 小, 仅为 $0.16 \mathrm{~nm}^{2[18]}$, 但是参与形成反胶团后, 仍起 到增大其油水界面积的作用, 即与 AOT 体系相比, 反胶团粒子半径增大. 粒子半径增大使反胶团界面 层易于形成双电层, 使带电粒子移动缓慢, 所以其电 导率值会变小.

加人非离子表面活性剂 Tween60 后, 对于 AOT 体系和 SDS 体系电导率的影响一样. 从上述分析可 知, 有正己醇作为助表面活性剂的 SDS 体系的反胶 团粒子半径比 $\mathrm{AOT}$ 体系的大, 造成带电粒子移动缓 慢, 所以与非离子表面活性剂 Tween60 混合后, 增 溶水量为 $W_{0, \text { max }}$ 时, 对于 SDS 体系, 随着阴离子表面 活性剂的摩尔分数增加, 电导率降低, 而对于 AOT 体系, 随着阴离子表面活性剂的摩尔分数增加, 电导 率增大.

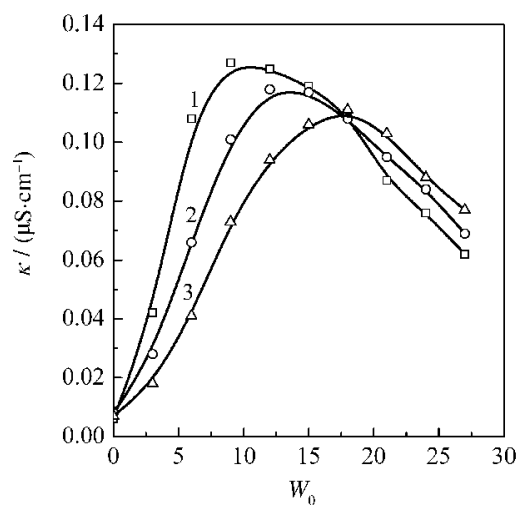

图 $5 \mathrm{SDS} / \mathrm{TrionX}-100 /$ 正己醇/环己烷/水体系的 $\kappa-W_{0}$ 图

Fig.5 Variations of $\kappa$ with $W_{0}$ in SDS/TrionX-100/ hexanol/cyclohexane/water system $x_{\text {sps: }}$ (1) 0.5, (2) 0.7, , (3) 0.9

\subsection{SDS/ TrionX-100/正己醇/环己烷/水体系}

SDS 与 Tween60 复配体系在正己醇和环己烷 中形成反胶团中的电导行为与 AOT 与 Tween60 复 配在非极性溶剂中的反胶团的电导行为存在着一定 的差异, 主要由于加人助表面活性剂醇的原因. 为了 更好地印证该结论,将非离子表面活性剂 Tween60 替换成 TrionX-100, 来研究其复配体系在正己醇和 环已烷混合溶剂中形成反胶团的电导行为. 图 5 中 仅考察了 $x_{\mathrm{SDS}} \geqslant 0.5$ 的情况, 可见其变化规律与 Tween60 体系类似, 随着离子型表面活性剂摩尔分 数的增大, $W_{0, \text { max }}$ 向 $W_{0}$ 增大的方向移动, 所对应的电 导率值也减小, 而且 $W_{0}$ 值较小时, 体系电导率值随 着 $x_{\mathrm{SDS}}$ 的增加而降低, 当 $W_{0}$ 值较大时, 体系电导率 值随着 $x_{\mathrm{SDS}}$ 的增加而增加, 与 SDS/TrionX-100/正己 醇/环己烷/水体系的现象完全一致, 这进一步证明了 SDS 和 AOT 体系现象的差异是由于助表面活性剂 醇的影响.

\section{3 结 论}

在 SDS/ Tween60/正己醇/环己烷/水体系中, 发 现与 SDS 单组分体系一样, 在电导率随增溶水量 $W_{0}$ 的关系曲线中, 电导率存在最大值, 所对应的增 溶水量为最大增溶水量 $W_{0, \max }$. 在复配体系中, 最大 增溶水量 $W_{0, \text { max }}$ 随着复配体系中 $x_{\mathrm{SDS}}$ 增大而向 $W_{0}$ 增 大的方向移动. 当 $x_{\mathrm{SDS}} \leqslant 0.5$ 时, 其对应的电导率值 却呈现出随着增溶水量的增大而增大, 当 $x_{\mathrm{SDS}} \geqslant 0.5$ 时,其随着增溶水量的增大而减小. 与 AOT/Tween60/ 环已烷/水体系进行了对比, 证明了在需要加人助表 面活性剂形成反胶团的 SDS 与非离子表面活性剂 复配体系中, 主要是由于醇的加人产生的影响. 在 
SDS/TrionX-100/正已醇/环已烷/水体系中的现象进

一步印证了该结论.

\section{References}

1 Eicke, H. F.; Borkovec, M.; Das-Gupta, B. J. Phys. Chem., 1989, 93: 314

2 Kallay, N.; Tomic, M.; Chittofrati, A. Colloid Polym. Sci., 1992, 207: 194

3 Franqueville, E.; Loutrari, H.; Mellou, F.; Stamatis, H.; Friboulet, A.; Kolisis, F. N. J. Mol. Catal. B-Enzym., 2003, 21: 15

4 Uskokovic, V.; Drofenik, M. Advances in Colloid and Interface Science, 2007, 133: 23

5 Hieda, J.; Saito, N.; Takai, O. Surface and Coatings Technology, 2008, 202: 5343

6 Lei, Z. L.; Wei, X. Y.; Bi, S. X.; He, R. J. Materials Letters, 2008 62: 3694

7 Yang, X. H.; Wu, Q. S.; Li, L.; Ding, Y. P.; Zhang, G. X. Colloids and Surfaces A-Physicochemical and Engineering Aspects, 2005, 264: 172

8 Hoefelmeyer, J. D.; Liu, H. J.; Somorjai, G. A.; Tilley, T. D J. Colloid Interface Sci., 2007, 309: 86

9 Liu, Y.; Dong, X. Y.; Sun, Y. Chinese Journal of Chemical
Engineering, 2008, 16(6): 949

10 Rajib, K.; Mitra, B.; Paul, K. Colloids and Surfaces APhysicochemical and Engineering Aspects, 2005, 252: 243

11 Chatterjee, S.; Mitra, R. K.; Paul, B. K.; Bhattacharya, S. C. J. Colloid Interface Sci., 2006, 298: 935

12 Liu, D. J.; Ma, J. M.; Cheng, H. M.; Zhao, Z. G. Colloids and Surfaces A-Physicochemical and Engineering Aspects, 1998, 135: 157

13 Liu, D. J.; Ma, J. M.; Cheng, H. M.; Zhao, Z. G. Colloids and Surfaces A-Physicochemical and Engineering Aspects, 1999, 148: 291

14 Eicke, H. F.; Thomas, H. Langmuir, 1999, 15: 400

15 Mitchell, D. J.; Ninham, B. W. J. Chem. Soc. Faraday Trans., 1981, 77: 601

16 Xiao, J. X.; Xiao, H.; Lan, T.; Bao, Y. X.; Zhao, Z. G. Acta Chim. Sin., 2004, 62(4): 351 [肖进新, 肖 寒, 蓝 亭, 暴艳霞, 赵振 国. 化学学报, 2004, 62(4): 351]

17 Zhou, G. W.; Li, G. Z.; Chen, W. J. Science in China Series B: Chemistry, 2001, 31(6): 508 [周国伟, 李干佐, 陈文君. 中国科 学 B 辑: 化学, 2001, 31(6): 508]

18 Biais, J.; Bodet, J. F.; Clin, B.; Lalanne, P.; Roux, D. J. Phys. Chem., 1986, 90: 5835 